INRA Prod. Anim., 2008, 21 (3), 269-276

\title{
Evolution des axes de recherche à I'INRA pour l'amélioration génétique du lapin de chair
}

\author{
H. GARREAU, J.-M. BRUN, M. THEAU-CLEMENT, G. BOLET
}

INRA, UR631 Amélioration Génétique des Animaux, F-31326 Castanet-Tolosan, France

Courriel :Herve.Garreau@toulouse.inra.fr

Jusqu'au début des années 2000, l'amélioration génétique du lapin de chair concernait essentiellement la prolificité dans les lignées maternelles et la vitesse de croissance dans les lignées paternelles. L'évolution du contexte sanitaire, économique et social a conduit les chercheurs de l'INRA à étudier la variabilité génétique de nouveaux caractères pour mieux prendre en compte la santé, la survie et l'adaptation des animaux à leur environnement.

Comme chez le porc et les espèces avicoles, les schémas d'amélioration génétique du lapin de chair sont organisés de manière pyramidale. Des firmes privées sélectionnent des lignées spécialisées paternelles et maternelles. Ces lignées sont ensuite multipliées pour proposer aux élevages de production des femelles croisées, qui seront accouplées ou le plus souvent inséminées, avec des mâles d'une lignée terminale pour produire les lapereaux de boucherie. Les objectifs de sélection sont un ensemble de caractères pour lesquels on cherche une amélioration de la valeur génétique additive des animaux d'une population. Les critères de sélection sont des caractères mesurables choisis en fonction des objectifs de sélection. Le choix des reproducteurs se fait sur une valeur appelée «indice synthétique de sélection» qui est une combinaison linéaire des valeurs génétiques des critères d'intérêt (critères de productivité et critères d'adaptation) avec des pondérations qui dépendent de l'importance accordée à chacun d'eux. Classiquement les objectifs de sélection des lignées paternelles sont la croissance et la qualité bouchère. Les principaux critères de sélection de ces lignées sont la vitesse de croissance post-sevrage ou le poids à âge type (Estany et al 1992, Lukefahr et al 1996, Larzul et al 2004) et, dans certains schémas de sélection, le rendement et l'adiposité de la carcasse (Garreau et al 2008a). L'objectif de sélection classique des lignées maternelles est la prolificité et les critères de sélection correspondants sont, principalement, la taille de la portée à la naissance ou au sevrage (Gómez et al 1996, Rochambeau 1998, Garreau et al 2004a)

La perception de l'élevage par le citoyen-consommateur a profondément évolué au cours des dernières années, avec de fortes attentes relatives à la santé publique, à l'environnement et au bien-être animal. L'évolution des objectifs et des critères de sélection doit désormais prendre en compte ces attentes, qui s'ajoutent à celles des professionnels : rentabilité de l'élevage, évolution des systèmes de production, avec la conduite en bande et la généralisation de l'insémination artificielle. La mortalité des femelles en reproduction (30\% en moyenne) et des jeunes $(25 \%$ en moyenne entre la naissance et la vente) pose des problèmes économiques et éthiques, qui ont conduit les généticiens à mettre en place des programmes d'amélioration génétique des aptitudes maternelles et de la longévité de la lapine. Les pathologies infectieuses comptent également parmi les problèmes majeurs de la filière. Les pertes économiques qui résultent de la mortalité, de performances zootechniques réduites et de l'usage d'antibiotiques réduisent le revenu de l'éleveur et posent le problème des résidus médicamenteux potentiels dans la viande, tout en nuisant à l'image du produit. La résistance aux maladies est ainsi devenue une thématique de recherche majeure des généticiens. Dans un contexte de forte inflation du prix des céréales qui grève lourdement le revenu des éleveurs, l'amélioration de l'efficacité alimentaire du jeune lapin à l'engraissement constitue également un objectif majeur. Par ailleurs, l'insémination artificielle de la lapine a fait son apparition dans les élevages français à la fin des années 80 . Ce mode de reproduction a permis la mise en place d'un nouveau système de production basé sur la «conduite en bande» et une meilleure organisation des élevages. Généralement, les éleveurs achètent la semence (mélanges hétérospermiques) dans l'un des 20 centres de production et réalisent eux-mêmes les inséminations. Plus de $80 \%$ des élevages rationnels français sont conduits en bande unique. Cette évolution a conduit à rechercher des voies d'amélioration génétique de la production spermatique des mâles. De plus, afin de diminuer l'utilisation des hormones pour l'induction de l'oestrus en insémination artificielle, de nouveaux programmes visent à étudier la variabilité génétique du comportement d'oestrus des lapines.

\section{1 / Les aptitudes mater- nelles}

\section{1 / Amélioration de la croissan- ce du jeune}

Comme chez le porc, la sélection pour la prolificité a conduit à une augmentation de la mortalité des jeunes sous la mère et à une réduction du poids au sevrage (Rochambeau 1998). Au début des années 2000, les éleveurs et les sélectionneurs ont donc souhaité modifier les objectifs de sélection des lignées maternelles pour améliorer la survie et le poids du jeune lapereau tout en maintenant l'intégrité de la femelle. L'environnement créé par la mère conditionne la survie et la croissance du jeune lapereau par de nombreux aspects : la mère intervient pendant la gestation en participant à la croissance embryonnaire et à la transmission des défenses immunitaires, puis en construisant un nid et enfin en allaitant ses petits pendant la phase post-partum. Le comportement maternel, autorisant la tétée, ainsi que la quantité et la qualité du colostrum et du lait ont une influen- 
Figure 1. Evolution génétique des effets directs et maternels du poids au sevrage pour les trois premières générations de sélection de la souche INRA 1777.

(g)

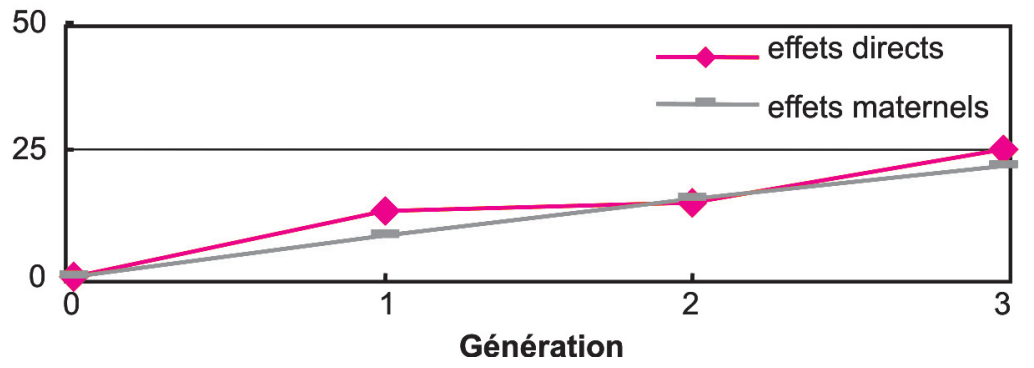

ce déterminante sur le poids des jeunes lapereaux. La lactation de la lapine dure en moyenne $35 \mathrm{j}$ avec un pic de lactation à $21 \mathrm{j}$ correspondant au moment où les lapereaux commencent à consommer de l'aliment solide. De la naissance au sevrage le poids de la portée passe de $600 \mathrm{~g}$ à $7500 \mathrm{~g}$ en moyenne soit respectivement $13 \%$ et $166 \%$ du poids adulte de la lapine $(4500 \mathrm{~g})$. Au pic de lactation, les besoins de lactation sont en moyenne trois fois plus élevés que les besoins d'entretien. Il est difficilement envisageable de mesurer en routine chacune des composantes des aptitudes maternelles (quantité et qualité du colostrum et du lait, comportement maternel) dans le cadre d'un programme de sélection. En revanche, il est relativement aisé de peser les lapereaux au sevrage.

Les modèles génétiques à effets directs et maternels s'appliquent à des caractères mesurés chez le jeune et dépendant plus ou moins fortement du milieu maternel (Laloë 1994). La part génétique d'une performance se décompose alors en un effet génétique direct (incidence du génotype de l'ani-

Figure 2. Différence entre les lignées homogène et hétérogène pour l'écart-type intraportée du poids à la naissance (en grammes).

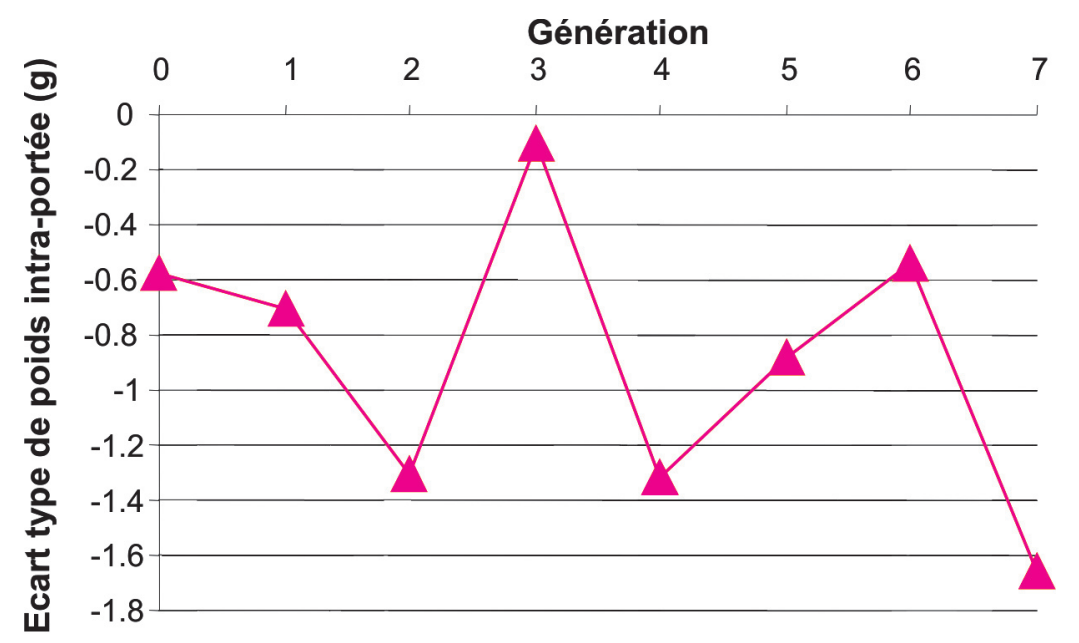

\section{2 / Amélioration de la survie périnatale par sélection pour l'homogénéité des poids de nais- sance}

La mortalité des jeunes lapereaux est associée à l'hétérogénéité des poids intra-portée. L'analyse du déterminisme génétique de la variabilité environnementale des caractères a fait l'objet de plusieurs études récentes. De nouveaux développements méthodologiques ont permis de mettre au point des modèles statistiques pour la mise en ouvre de la sélection canalisante (San Cristobal-Gaudy et al 1998). Cette méthode, qui permet de sélectionner une population pour un niveau de performance optimal en réduisant la variabilité du caractère autour de l'optimum, a été appliquée à l'INRA avec la mise en place d'une expérience de sélection divergente sur la variabilité des poids à la naissance des lapereaux d'une lignée commerciale (Grimaud Frères Sélection). L'objectif était d'obtenir une lignée avec des poids de lapereaux homogènes, une autre lignée avec des poids de lapereaux hétérogènes. Après sept générations de sélection, une différence très significative est obtenue entre les deux lignées pour l'écart-type intra-portée du poids à la naissance $(1,6 \mathrm{~g}$, soit $19 \%$ de la moyenne du caractère (figure 2), sans que la moyenne du poids à la naissance ne soit affectée de façon significative (Garreau et al 2004b, 2008b, Bolet et al 2007a, 2008). On observe également des réponses corrélées favorables dans la lignée homogène sur l'écart-type intra-portée du poids au sevrage (9 g, soit $12 \%$ de la moyenne du caractère), sur la mortinatalité et sur la mortalité naissance-sevrage (figure 3). Des mesures des caractéristiques du tractus génital ont également été réalisées, montrant que les cornes utérines des lapines de la lignée homogène sont plus longues et plus étirables (figure 4).

Cette méthode de sélection a été mise en oeuvre dans une lignée commerciale dans le but d'améliorer génétiquement les aptitudes maternelles à la survie du jeune. La poursuite de l'expérimentation permettra de mieux caractériser la réponse à la sélection par des mesures de croissance et de survie embryonnaire et l'étude anatomique des cornes utérines en liaison avec l'activité hormonale. 
Figure 3. Différence entre les lignées homogène et hétérogène pour le taux de mortinatalité et de mortalité naissance-sevrage (en \%).

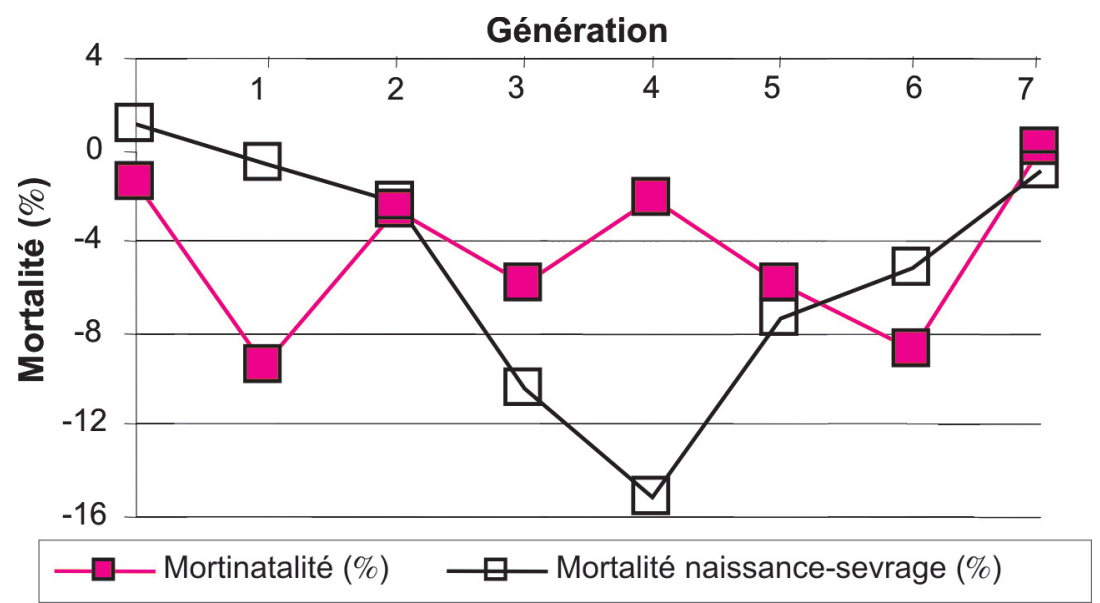

Figure 4. Longueur initiale (LO) et élongation de la corne utérine après ajout d'un poids de $50 \mathrm{~g}(L 1)$ ou $70 \mathrm{~g}(\mathrm{L2})$ dans les lignées homogène et hétérogène en 3ème (4a) et sixième $(4 b)$ générations.
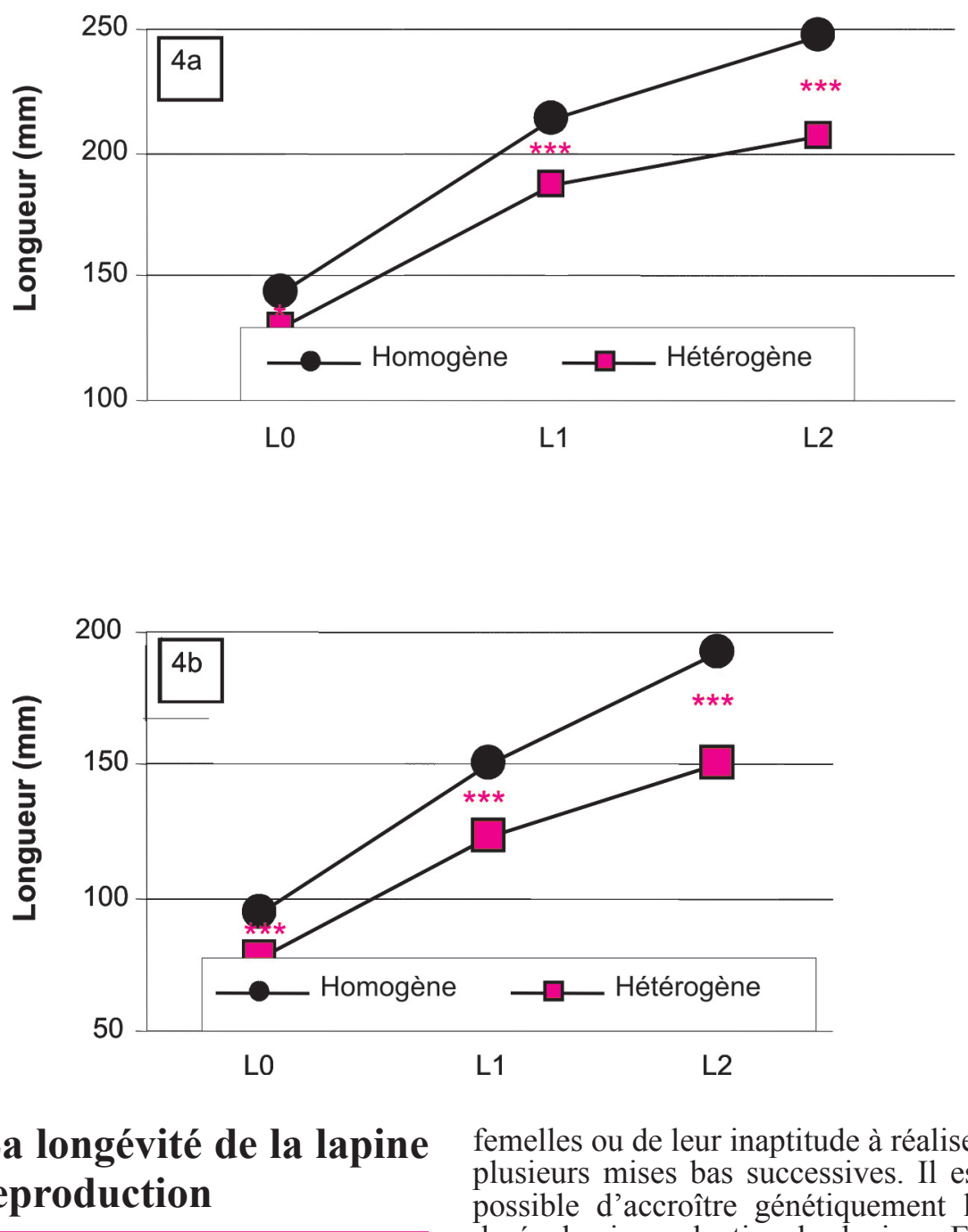

Dans les élevages de production, la mortalité des reproductrices est de $30 \%$ en moyenne. Les autres causes de fonte de cheptel sont variées, mais dépendent souvent d'une baisse de fertilité des
2004, Piles et al 2006, Theilgaard et al 2007).

Pour vérifier l'efficacité de ce type de sélection, pour analyser les conséquences sur les autres caractères de reproduction et pour comprendre les mécanismes physiologiques sous-jacents, une sélection divergente pour la longévité fonctionnelle, basée sur un index estimé grâce aux techniques d'analyse de survie, a été mise en place à l'INRA en 2003 (Garreau et al 2007, Larzul et al 2007). Sachant que les femelles sont conduites en bande, le critère retenu pour estimer la durée de vie productive a été le nombre d'Inséminations Artificielles (IA) auquel la lapine a été soumise dans sa carrière, sans réaliser d'éliminations pour cause d'infertilité. L'analyse de la longévité et des performances de reproduction de la seconde génération de femelles a permis d'estimer la réponse à la sélection. Les courbes de survie sont représentées à la figure 5 par lignée et par élevage. L'effet de la lignée était très significatif $(\mathrm{p}=0,001)$ et la différence de longévité entre les 2 lignées est de 0,92 IA soit $39 \mathrm{j}$. Les deux lignées ne diffèrent par contre pas pour le nombre de nés vivants ou sevrés par mise bas comme attendu sur la base des relations génétiques entre productivité et longévité, mal connues, mais semble-t-il relativement faibles. Ainsi, Tudela et al (2003) ont montré que la sélection sur la taille de portée n'accroissait pas le risque de réforme. Sanchez et al (2004) ont quant à eux obtenu des corrélations génétiques proches de 0 , entre la longévité et le nombre de nés vivants et de sevrés.

Des mesures de bilan énergétique sur les femelles de la seconde génération sont en cours d'analyse et doivent permettre de mieux comprendre les mécanismes biologiques impliqués dans la durée de la carrière reproductive. Le transfert de cette méthode de sélection vers la profession est en cours.

\section{3 / Résistance aux maladies}

Parmi les maladies susceptibles d'affecter le lapin, seules quelques unes, peuvent occasionner de lourdes pertes au sein du troupeau. Il s'agit des affections de l'estomac et de l'intestin et de certaines maladies infectieuses (pasteurellose, myxomatose, VHD). L'étude de la résistance aux maladies présente de nombreuses contraintes : utilisation de bâtiments expérimentaux protégés pour l'infection expérimentale, coût et 
Figure 5. Courbes de survie des femelles de la lignée haute (+) et de la lignée basse (-) de l'expérience de sélection divergente pour la longévité des femelles INRA 1077 en reproduction.

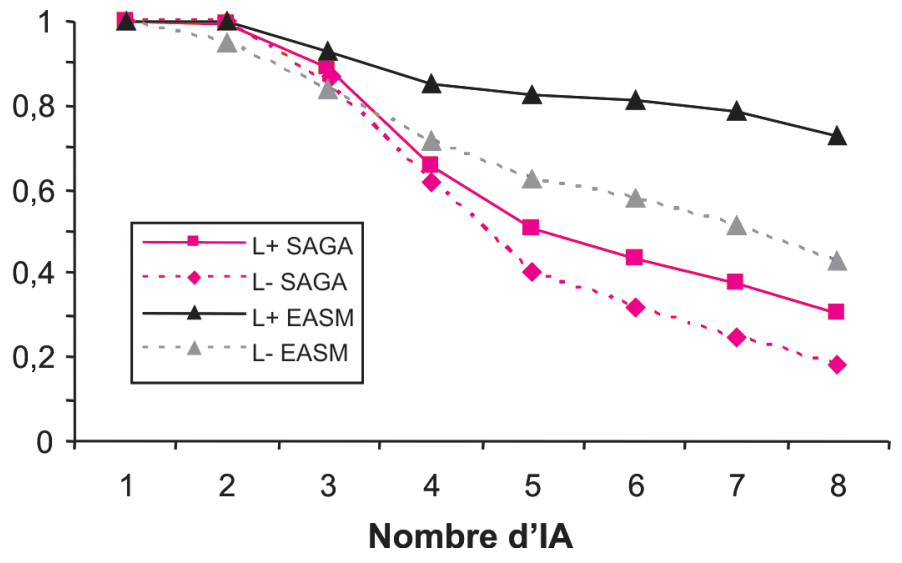

difficulté de la mesure de la réponse à l'infection naturelle ou expérimentale. Elle est de ce fait réservée aux pathologies ayant un fort impact sur les résultats économiques et/ou pour lesquelles les moyens de lutte classiques (prophylaxie, vaccins) sont peu efficaces ou mal acceptés par le consommateur. L'INRA étudie actuellement le déterminisme génétique de deux pathologies majeures du lapin : l'entérocolite épizootique et la pasteurellose. La localisation et l'identification des gènes associés à une résistance accrue apparaissent comme un moyen particulièrement prometteur pour intégrer ces caractères dans les objectifs de sélection.

\section{1 / La résistance à l'entérocoli- te épizootique et aux troubles digestifs non spécifiques}

L'Entérocolite Epizootique du Lapin (EEL) est un syndrome gastro-intestinal émergent qui est apparu à la fin de l'année 96 dans quelques élevages de l'ouest de la France, puis s'est étendu aux autres régions et à d'autres pays européens au cours des années 97 et 98. Cette maladie se caractérise principalement par une distension de l'abdomen, suivie d'une perte d'appétit, et par de fortes mortalités (30 à $80 \%$ au début de l'épizootie). Malgré les progrès réalisés pour la maîtrise de la mortalité, notamment en associant l'amélioration de l'environnement d'élevage, l'antibiothérapie et le rationnement des animaux, les pathologies digestives demeurent la principale cause de mortalité en engraissement. A ce jour l'agent responsable de l'EEL n'a toujours pas été identifié.

Dans une première étude, Rochambeau et al (2006) ont analysé la variabilité génétique pour la sensibilité aux troubles digestifs de quatre types génétiques de lapins en utilisant trois modèles de reproduction de la maladie : l'infection expérimentale par des coccidies, la distribution d'un aliment déficient en fibres et enfin une inoculation avec l'inoculum mis au point par l'INRA de Tours et permettant de reproduire l'EEL. L'objectif de l'expérience était de vérifier s'il existait un déterminisme génétique commun pour les trois modèles et de vérifier l'hypothèse d'une résistance d'organe indépendante du facteur d'agression. Une variabilité significative entre pères des lapereaux de l'expérience a été montrée pour la mortalité et la morbidité avec les deux premiers modèles, mais seulement pour la morbidité avec le modèle EEL. Les classements des pères pour les trois modèles étaient faiblement corrélés, démontrant un déterminisme génétique de la résistance assez différent selon le type d'agression. Dans une seconde expérience, la réponse à l'inoculation de l'EEL a été mesurée sur deux bandes de 330 lapereaux. Les héritabilités estimées pour la mortalité, l'indice de croissance normale et la résilience étaient respectivement de $0,05,0,07$ et 0,28 (Garreau et al 2006).

Compte tenu des faibles valeurs d'héritabilité obtenues et des contraintes liées à l'infection expérimentale, une autre méthode d'étude de la résistance aux troubles digestifs a été récemment proposée : un index binaire (présence ou absence de troubles digestifs) a été construit à partir d'enregistrements sanitaires pratiqués en routine dans une lignée mâle (AGP39) du sélectionneur Grimaud Frères Sélection. La totalité des animaux candidats à la sélection depuis 1998 (46 000 animaux à ce jour) a été inspectée après la pesée, à $63 \mathrm{j}$ d'âge, ou à la mort de l'animal si elle intervenait avant la pesée. Les paramè- tres génétiques de cet index et des autres caractères de l'objectif de sélection (poids à $63 \mathrm{j}$, rendement de la carcasse, adiposité de la carcasse) ont été estimés (Garreau et al 2008a). Les héritabilités estimées étaient respectivement de $0,08,0,36,0,24$ et 0,64 pour l'index de résistance, le poids à $63 \mathrm{j}$, le rendement de carcasse et l'adiposité de la carcasse. Les corrélations génétiques entre l'index de résistance et les autres caractères sélectionnés étaient toutes négatives et donc favorables (de - 0,07 à - 0,34).

Le sélectionneur Grimaud Frères Sélection réalise actuellement une validation expérimentale de cette méthode avec l'appui de l'INRA pour introduire cet index dans l'objectif de sélection d'une de ses lignées mâles.

\section{2 / La résistance à la pasteurel- lose}

Bien qu'il n'existe pas d'étude épidémiologique précise, la pasteurellose est reconnue comme l'une des maladies les plus fréquentes et les plus graves chez le lapin, aussi bien en élevage qu'en animalerie de laboratoire (Coudert 2004). Provoquant des affections des organes respiratoires et touchant également d'autres organes (abcès cutanés, mammite, vaginite, métrite), elle est la principale cause de réforme sanitaire des femelles. La première estimation des paramètres génétiques de la résistance à la pasteurellose a été publiée par Baselga et al (1988). L'héritabilité du critère, basé sur la présence de lésions pulmonaires après autopsie, était comprise entre 0,12 et 0,28 . A partir de 2002, une collaboration entre la SAGA et le Commonwealth Scientific and Industrial Research Organisation (CSIRO) d'Armidale en Australie a permis d'estimer les paramètres génétiques de la résistance à Pasteurella Multocida à partir de symptômes extérieurs relevés pendant la période de croissance de 5 à 12 semaines d'âge (Eady et al 2004, 2007). Un index de l'incidence de la maladie a été construit pour chaque lapin. L'héritabilité de cet index est de 0,09. Il est aujourd'hui utilisé pour sélectionner les animaux du noyau de sélection du CSIRO pour la résistance à la pasteurellose. Cette méthode n'est toutefois pas applicable aux élevages de sélection français car, en raison de la surprotection des noyaux de sélection, l'incidence de la maladie y est très faible. Par ailleurs, les élevages de production et de multiplication n'enregistrent pas les états sanitaires individuels des animaux. 
Répondant à la demande des principaux acteurs de la profession (Interprofession, syndicats des sélectionneurs, fédération des éleveurs), l'INRA a proposé un projet visant à mettre au point un modèle de reproduction expérimentale de la maladie pour étudier le déterminisme génétique de la résistance. Cette épreuve sera ensuite utilisée pour estimer la variabilité familiale et raciale de la résistance à la pasteurellose. L'objectif est d'identifier des marqueurs moléculaires associés à la résistance à la maladie pour une sélection assistée par marqueur ou une sélection génomique dans les noyaux de sélection.

\section{4 / L'efficacité alimentaire}

L'alimentation représente $60 \%$ des coûts de production de l'élevage du lapin de chair et l'augmentation brutale du coût des matières premières survenue en 2007 compromet fortement la rentabilité des élevages. L'amélioration de l'efficacité alimentaire est donc de plus en plus importante pour assurer la compétitivité de la filière cunicole par rapport aux autres filières viande. Elle doit également permettre la diminution des rejets animaux et la réduction de la mortalité en engraissement. Le rationnement des animaux en engraissement, largement appliquée dans les élevages français pour limiter les troubles digestifs, permet d'améliorer l'efficacité alimentaire (Gidenne et al 2003). L'amélioration de ce caractère peut également se faire par la voie génétique. La dépense énergétique d'un animal se décompose en deux parties : la dépense affectée aux besoins de production et la dépense affectée aux besoins d'entretien. Pendant la période de croissance, la dépense pour les besoins de production dépend directement du gain de poids vif et de la composition de ce gain de poids (répartition protéines/lipides). Les variations individuelles des besoins de production et d'entretien sont intégrées dans la consommation moyenne résiduelle journalière, définie comme la quantité d'aliment consommée en plus ou en moins par rapport aux besoins théoriques pour l'entretien et la production (Kennedy et al 1993). Une première stratégie consiste à améliorer indirectement l'efficacité alimentaire en sélectionnant la vitesse de croissance. Une seconde stratégie consiste à sélectionner sur un critère tel que l'Indice de Consommation (IC). Chez le lapin, les valeurs d'héritabilité de l'IC récentes reportées dans la littérature sont moyennes $(0,31$ et 0,25 Piles et al 2004 ; 0,27 Larzul et Rochambeau 2005) et laissent supposer qu'une sélection directe sur ce critère serait efficace. Cependant, compte tenu de la nature de ce critère (rapport entre deux caractères), il est difficile de savoir dans quelle mesure les deux variables impliquées (la quantité d'aliment ingéré et le gain de poids) sont modifiées par la sélection. Une troisième stratégie consiste à exploiter la variabilité génétique de la dépense énergétique pour les besoins d'entretien en sélectionnant la consommation moyenne résiduelle journalière. Une expérience de sélection portant sur 6 générations a débuté en 2007 à l'INRA de Toulouse. L'objectif est de comparer deux méthodes de sélection avec utilisation d'une population témoin non sélectionnée :

- une sélection sur la consommation moyenne résiduelle journalière ;

- une sélection sur la vitesse de croissance en régime alimentaire rationné.

Dans le premier cas, l'idée est d'améliorer l'efficacité alimentaire, en diminuant la consommation. Ce type de sélection a déjà été mis en œuvre chez les bovins, les ovins, les porcs, ainsi que chez le lapin (Larzul et Rochambeau 2005) avec des résultats probants. L'efficacité alimentaire est bien améliorée tandis que la vitesse de croissance et la composition corporelle sont peu affectées.

Pour la seconde méthode, il s'agit d'améliorer l'efficacité alimentaire en augmentant la vitesse de croissance tout en contrôlant le niveau d'ingestion. Elle est déjà indirectement réalisée par les sélectionneurs de lapins qui, essentiellement pour des raisons sanitaires, pratiquent un rationnement des lapereaux. Outre les critères d'efficacité alimentaire, la comparaison des deux lignées portera sur des critères de santé des animaux, de croissance, de comportement alimentaire et de niveau d'ingestion, de digestion, d'activité microbienne et de flore digestive, de qualité des carcasses et de qualité de viande. La recherche de marqueurs génétiques pour la mise en place d'une sélection assistée par marqueur est particulièrement intéressante pour ce type de caractère en raison de la lourdeur des mesures de consommation individuelle d'aliment.

\section{5 / La production sperma- tique des mâles et la fertili- té des femelles}

Différentes études ont mis en évidence des effets génétiques sur la production spermatique tant dans ses aspects quantitatifs que qualitatifs (TheauClément et al 1999, 2007, Brun et al 2002a, 2006). Les sélectionneurs s'interrogent donc sur la pertinence d'intégrer des indicateurs de la production spermatique parmi les critères de sélection des souches de mâles de croisement terminal. Pour répondre à ce questionnement, une expérience en cours a pour objectif d'estimer les paramètres génétiques des caractéristiques de la semence et d'étudier leurs relations avec la fécondité après l'insémination, encore mal connues chez cette espèce (Brun et al 2002b).

Dans les élevages français, les résultats de fertilité sont de bon niveau ( $77 \%$ en moyenne), cependant les éleveurs utilisent diverses méthodes, notamment des méthodes hormonales (en particulier eCG) pour induire la réceptivité des lapines au moment de l'insémination. En effet, les lapines qui ne sont pas sexuellement réceptives (en anoestrus) au moment de l'insémination ont une productivité trois fois plus faible que les femelles réceptives et sont une source d'hétérogénéité des performances de reproduction (TheauClément 2007). Dans un contexte de recherche de systèmes d'élevage plus durables, un programme vise à rechercher des alternatives à l'utilisation d'hormones. Une première solution concerne la recherche de facteurs de milieu (photopériode...) ou de techniques d'élevage (courte séparation de la mère et sa portée...) généralement regroupés sous le terme de «biostimulations» (Theau-Clément et al 2005). Une deuxième solution consiste à exploiter la voie génétique pour favoriser l'induction de l'oestrus et en conséquence améliorer et homogénéiser les performances de reproduction dans les élevages. Cette étude débutera en 2008 , par une meilleure caractérisation de la réceptivité, incluant la mesure de la répétabilité de ce comportement d'une période post-partum à l'autre.

\section{Perspectives et conclusion}

Jusqu'à présent, les outils de la génétique moléculaire n'ont pas été utilisés pour la sélection du lapin de chair. Les 
effets de quelques gènes candidats ont été décrits par plusieurs équipes de recherche : Bolet et al (2007b) ont mis en évidence un effet significatif du gène de la caséine Kappa sur la prolificité. Dans deux lignées sélectionnées de façon divergente pour la capacité utérine, Peiro et al (2006) et Merchan et al (2006) ont démontré l'effet de deux gènes candidats sur le nombre d'embryons et la taille de portée à la naissance : le gène codant pour le récepteur de la progestérone et le gène de l'oviductine. Les données et les outils de la génomique pour le lapin ont largement progressé au cours des cinq dernières années. Le lapin bénéficie maintenant d'une carte génétique, de plusieurs banques de BAC, d'une carte cytogénétique de gènes et de marqueurs microsatellites (Chantry-
Darmon et al 2006). Dans le cadre du Mamalian Genome project, un programme de séquençage complet du génome du lapin sera achevé en 2009 par le Broad Institute (Boston, USA) en collaboration avec des laboratoires de l'INRA et d'autres laboratoires européens (Rogel-Gaillard et al 2008), donnant au lapin le statut de nouvelle espèce séquencée. De nouvelles ressources génomiques seront alors produites : des banques d'ADNc de pleine longueur permettront l'expression de protéines et la production d'anticorps. L'ADN de lapins appartenant à 4 races et de deux lapins sauvages sera également entièrement séquencé afin d'identifier des polymorphismes nucléotidiques et de produire des marqueurs SNP. Les marqueurs SNP (pour «Single Nucleotide Polymorphism» ou varia- tion d'une paire de bases du génome) sont présents en très grande quantité sur le génome (plusieurs millions) et autorisent les méthodes de génotypage à haut débit (puces avec génotypage de plusieurs milliers de SNP simultanément).

L'information apportée par ces nouveaux outils ouvre la voie à de nouvelles méthodes de sélection (sélection assistée par marqueur, sélection génomique) qui se révèlent particulièrement intéressantes pour l'amélioration génétique des caractères dont la mesure est souvent coûteuse ou contraignante. Ces méthodes ne présenteront toutefois un réel intérêt qu'avec de faibles coûts de génotypage, compte tenu de la faible valeur commerciale des reproducteurs comparativement à d'autres espèces.

\section{Références}

Baselga M., Deltoro J., Camacho J., Blasco A., 1988. Genetic analysis on lung injury in four strains of meat rabbit. Proc. $4^{\text {th }}$ World Rabbit Congr., Budapest, Hungary, 1, 120-127.

Bolet G., Garreau H., Joly T., Theau-Clément M., Falières J., Hurtaud J., Bodin L., 2007a. Genetic homogenization of birth weight in rabbits: indirect selection response for uterine horns characteristics. Livest. Sci., 111, 28-32.

Bolet G., Devinoy E., Virag G., Harsanyi I., Bosze Z., 2007b. Association between litter size and the $\mathrm{k}$ casein genotype in the INRA rabbit lines. World Rabbit Sci., 15, 147-150.

Bolet G., Garreau H., Hurtaud J., Saleil G., Esparbié J., Falières J., Theau-Clément M. Bodin L., 2008. Canalising selection on within litter variability of birth weight in rabbits: responses to selection and characteristics of the uterus of the does. Proc. 9th World Rabbit Congr., June 10-13, Verona, Italy, 51-55.

Brun J.M., Theau-Clément, M., Bolet, G. 2002a. Evidence for heterosis and maternal effects on rabbit semen characteristics. Anim. Res., 51, 433-442.

Brun J.M., Theau-Clément, M., Bolet, G. $2002 \mathrm{~b}$. The relationship between rabbit semen characteristics and reproductive performance after artificial insemination. Anim. Reprod. Sci., 70, 139-149.

Brun J. M., Theau-Clément M., Esparbié J., Falières J., Saleil G., Larzul C., 2006. Semen production in two rabbit lines divergently selec-ted for 63-d body weight. Theriogenology, 66, 2165 2172.

Chantry-Darmon C., Urien C., Rochambeau H. de, Allain D., Pena B., Hayes H., Grohs C.P., Cribiu E., Deretz-Picoulet S., Larzul C., Save J. C., Neau A., Chardon P., Rogel-Gaillard C. 2006. A first-generation microsatellite-based integrated genetic and cytogenetic map for the European rabbit (Oryctolagus cuniculus) and localization of angora and albino. Anim. Genet., 37, 335-341.

Coudert P., 2004. Pasteurelloses du lapin. Cuniculture, 31, 49-51.
Ducrocq V., Sölkner J., 1998. The survival kit V3.0, a package for large analyses of survival data. Proc. $6^{\text {th }}$ World. Congr. Appl. Livest. Prod., Armidale, Australie, 27, 447-450.

Eady S.J., Garreau H., Hurtaud J., 2004. Heritability of resistance to bacterial infection in commercial meat rabbit populations. Proc. $8^{\text {th }}$ World Rabbit Congr., Puebla, Mexico, 1, 51-56.

Eady S.J., Garreau H., Gilmour A.R., 2007. Heritability of resistance to bacterial infection in meat rabbits. Livest. Sci., 112, 90-98.

Estany J., Camacho J., Baselga M., Blasco A., 1992. Selection response of growth rate in rabbits for meat production. Genet. Sel. Evol., 24, 527-537.

Garreau H., Rochambeau H. de, 2003. La sélection des qualités maternelles pour la croissance du lapereau. 10 èmes Journ. Rech. Cunicole, 19-20 nov., Paris, France, 61-64.

Garreau H., Piles M., Larzul C., Baselga M., Rochambeau H. de, 2004a. Selection of maternal lines: last results and prospects. Proc. $8^{\text {th }}$ World Rabbit Congr., September 7-10, Puebla, Mexico, 14-25.

Garreau H., San Cristobal M., Hurtaud J., Bodin L., Ros M., Robert-Granie C., Saleil G., Bolet G., 2004b. Can we select on within litter homogeneity in rabbit birth weight? A divergent selection experiment. Proc. $8^{\text {th }}$ World Rabbit Congr., September 7-10, Puebla, Mexico, 63-68.

Garreau H., Duzert R., Tudela F., Baillot C. Ruesche J., Grauby G., Lille-Larroucau C., Rochambeau H. de, 2005. Gestion et sélection de la souche INRA 1777 : Résultats de trois générations de sélection, 11 èmes Journ. Rech. Cunicole, 19-20 nov., Paris, France, 19-22.

Garreau H., Licois D., Rupp R., Rochambeau H. de, 2006. Genetic variability of the resistance to Epizootic Rabbit Enteropathy (ERE): new results. Proc. $8^{\text {th }}$ World. Congr. Appl. Livest. Prod., 13-18 August, Belo Horizonte, Brazil, 4p.

Garreau H., Ducrocq V., Tudela F., Saleil G., Esparbié J., Juin H., Lamothe E., Sordello J.J.,
Larzul C., 2007. Sélection divergente pour la longévité de la lapine en reproduction. 12 èmes Journ. Rech. Cunicole, 27-28 nov., Le Mans, France, 129-132.

Garreau H, Eady S.J., Hurtaud J., Legarra A., 2008a. Genetic parameters of production traits and resistance to digestive disorders in a commercial rabbit population. Proc. $9^{\text {th }}$ World Rabbit Congr., June 10-13, Verona, Italy, 61-65.

Garreau H., Bolet G., Larzul C., Robert-Granié C., Saleil G., SanCristobal M., Bodin L., 2008b. Results of four generations of a canalising selection for rabbit birth weight. Livest. Sci., sous presse.

Gidenne T., Feugier A., Jehl N., Arveux P., Boisot P., Briens C., Corrent E., Fortune H., Montessuy S., Verdelhan S., 2003. Un rationnement alimentaire quantitatif postsevrage permet de réduire la fréquence des diarrhées, sans dégradation importante des performances de croissance : résultats d'une étude multi-site. 10 èmes Journ. Rech. Cunicole, Paris, France, 29-32.

Gómez E.A., Rafel O., Ramón J., Baselga M. 1996. A genetic study of a line selected on litter size at weaning. Proc. $6^{\text {th }}$ World Rabbit Congr., July 9-12, Toulouse, France, 2, 289-292.

Kennedy B.W., van der Werf J.H.J, Meuwissen T.H.E., 1993. Genetic and statistical properties of residual feed intake. J. Anim. Sci. 71, 3239-3250.

Laloe D., 1994. Application du modèle animal aux bovins allaitants. In : Séminaire modèle animal, INRA, Département de Génétique Animales, 26-29 septembre, La Colle sur Loup, France, 91-98.

Larzul C., Rochambeau H. de, 2005. Selection for residual feed consumption in the rabbit. Livest. Prod. Sci. 95, 67-72.

Larzul C., Gondret F., Combes S. Rochambeau H de, 2004. Divergent selection on 63 -day body weight in the rabbit: response on growth, carcass and muscle traits, Genet. Sel. Evol., 37, 105-122. 
Larzul C., Garreau H., Ducrocq V., 2007. Divergent selection for length of productive life in rabbit. $58^{\text {th }}$ Ann. Meet. Eur. Ass. Anim. Prod., Dublin, Ireland, August 26-29, Session 10, Theatre 13, Book of Abstracts, 57.

Lukefahr S.D., Odi H.B., Atakora J.K.A., 1996. Mass selection for 70 body weight in rabbits. J. Anim. Sci., 74, 1481-1489.

Merchán M., Peiró R., Argente M.J. García M.L., Agea I., Santacreu M.A., Blasco A., Folch J.M., 2006. Candidate genes for reproductive traits in rabbits: I. Oviductin gene. Proc. $8^{\text {th }}$ World. Congr. Appl. Livest. Prod., 13-18 August 2006, Belo Horizonte, Brazil. 4p.

Rogel-Gaillard C., Chantry-Darmon C., Hayes H., 2008. Les données récentes sur le génome du lapin. Biofutur, 287, 28-31.

Peiró R., Merchán M., Santacreu M.A., Argente M.J. García M.L., Agea I., Folch J.M., Blasco A., 2006. Candidate genes for reproductive traits in rabbits: II. Progesterone receptor gene. Proc. $8^{\text {th }}$ World. Congr. Appl. Livest. Prod., 13-18 August, Belo Horizonte, Brazil, $4 p$.

Piles M., Gomez E.A., Rafel O., Ramon J., Blasco A., 2004. Elliptical selection experiment for the estimation of genetic parameters of the growth rate and feed conversion ratio in rabbits. J. Anim. Sci., 82, 654-660.
Piles M., Garreau H., Rafel O., Larzul C., Ramon J., Ducrocq V., 2006. Survival analysis in two lines of rabbits selected for reproductive traits. J. Anim. Sci., 84, 1658-1665.

Rochambeau H. de, 1998. La femelle parentale issue des souches expérimentales de l'INRA évolutions génétiques et perspectives. 7èmes Journ. Rech. Cunicole, Lyon, France, 3-14.

Rochambeau H. de, Licois D., Gidenne T. Verdelhan S., Coudert P., Elsen J. M., 2006. Genetic variability of the resistance for three types of enteropathy in the growing rabbit. Livest. Sci., 101, 110-115.

Sánchez J.P., Peiro R., Silvestre M.A., Baselga M., 2004. Analysis of factors influencing longevity of rabbit does. Livest. Prod. Sci., 90, 227-234.

San Cristobal-Gaudy M., Elsen J.M., Bodin L., Chevalet C., 1998. Prediction of the response to a selection for canalisation of a continuous trait in animal breeding, Genet. Sel. Evol, 30, 423-451.

Theau-Clément M., 2007. Preparation of the rabbit doe to insemination: A review. World Rabbit Sci., 15, 61-80.

Theau-Clément M., Brun J.M., Bolet G., Esparbié J., Falières J., 1999. Constitution d'une souche synthétique à l'INRA : 2. Comparaison des caractéristiques biologiques de la semence des mâles des deux souches de base et de leurs croisements réciproques. 7 èmes Journ. Rech. Cunicole, Lyon, France, 127-130.

Theau-Clément M., Boiti C., Bonanno A. Eiben Cs, Maertens L., Szendro Zs., 2005. Alternative methods for oestrous synchronisation of lactating rabbit does. In: Recent advances in rabbit sciences. Maertens L., Coudert P. (Eds), Melle, Belgique, 21-37.

Theau-Clément M., Brun J.M., Esparbié J., Falières J., Garvanèse J., Lamothe E., Larzul C., Milcent N., Saleil G., 2007. Une sélection pour la vitesse de croissance influence-t-elle la fécondance de la semence de lapins ? 12 èmes Journ. Rech. Cunicole, 27-28 nov., Le Mans, France, 53-56.

Theilgaard P., Sánchez J.P., Pascual J.J., Berg P., Friggens N., Baselga M., 2007. Late reproductive senescence in a rabbit line hyper selected for reproductive longevity, and its association with body reserves. Genet. Sel. Evol., 39, 207223.

Tudela F, Hurtaud J, Garreau G, De Rochambeau H., 2003. Comparaison des performances zootechniques de femelles parentales issues d'une souche témoin et d'une souche sélectionnée sur la productivité numérique. 10 èmes Journ. Rech. Cunicole, Paris, France, 5356.

\section{Résumé}

L'évolution du contexte sanitaire, économique et social de la production de lapin de chair a conduit les chercheurs de l'INRA à étudier la sélection de nouveaux caractères pour mieux répondre aux attentes des professionnels mais également du citoyen-consommateur, en particulier dans le domaine de la santé et de la longévité des animaux. Ces études reposent principalement sur la mise en place d'expériences mais également sur l'analyse de données de sélectionneurs privés et donnent lieu, après validation, à des applications dans les schémas de sélection commerciaux. Pour réduire la mortalité des jeunes, les chercheurs privilégient l'amélioration des aptitudes maternelles : l'application d'un modèle à effets génétiques directs et maternels pour le poids au sevrage a ainsi permis d'améliorer les aptitudes maternelles pour ce caractère. Une expérience de sélection canalisante pour l'homogénéité des poids de naissance intra-portée a permis d'améliorer la survie périnatale du lapereau. L'amélioration de la longévité de la femelle en reproduction est rendue possible par la mise en ouvre des méthodes d'analyse de survie. La résistance génétique aux maladies, principalement l'entérocolite épizootique et la pasteurellose, est explorée pour réduire les pertes économiques mais également pour améliorer le bien-être des animaux et réduire les risques sanitaires liés à l'utilisation des antibiotiques. Pour améliorer l'efficacité alimentaire du jeune en engraissement et ainsi réduire les coûts alimentaires qui grèvent lourdement le revenu de l'éleveur, deux critères de sélection sont comparées : la consommation résiduelle et la vitesse de croissance en régime alimentaire rationné. Afin de diminuer l'utilisation des hormones pour l'induction de l'oestrus, de nouveaux programmes visent à étudier la variabilité génétique du comportement d'oestrus des lapines.

\section{Abstract}

\section{Evolution of research axes at INRA for the genetic improvement of meat rabbits}

The increased emphasis on triple bottom line reporting for agricultural businesses (economic, social and environmental) has led the genetic research program for meat rabbits to focus on new selection traits that improve animal health and welfare and moderate reliance on drugs. These studies are mainly based on experiments but also on analyses of data of private breeding companies and have led to applications in commercial breeding schemes after validation. In order to reduce mortality in young rabbits, scientists have been studying the maternal ability of the doe. The use of a complex genetic animal model including direct and maternal effects has made it possible to improve the maternal ability of does, resulting in improved survival and growth of young rabbits. In addition, an experiment of canalising selection has demonstrated that the survival of young rabbits is improved with selection for homogeneity of birth weight. An increase in longevity of breeding does has been demonstrated by the implementation of survival analysis. Genetic resistance to disease, mainly epizootic enterocolitis and pasteurellosis, has been investigated in order to reduce economic losses but also to improve animal welfare and to reduce risk due to the use of antibiotics. To improve feed efficiency and to reduce feeding costs, which are a major determinant of farmers' income, two selection criteria have been evaluated for possible inclusion in breeding programs: residual consumption and growth rate on a restricted food ration. In order to reduce the use of hormones for oestrus induction, new programs have commenced to study the genetic variability of oestrus behaviour in does.

GARREAU H., BRUN J.-M., THEAU-CLEMENT M., BOLET G., 2008. Evolution des axes de recherche à l'INRA pour l'amélioration génétique du lapin de chair. INRA Prod. Anim., 21, 269-276. 
